\title{
Design of a Remote Monitoring System for the Management of the Information on the Maintenance of Maneuvering Communication Lines
}

\author{
Yin Shu Hua*1, a , Lei Hü ${ }^{2, b}$, Ren Jia ${ }^{3, c}$ \\ ${ }^{1}$ Xi'an fanyi university, Xi'an, China \\ Shuhua_y@126.com
}

Keywords: Communication Line; Maintenance; Surveillance Management; MSP430F149; GSM

\begin{abstract}
Aiming at the special management requirements for maintaining communications lines, this paper develops a remote monitoring system for managing the information regarding the maintenance by considering the condition that maintenance workers from grass-roots maneuvering affiliations are dispersed. Based on the required functions, the modular structures are designed by using a MSP430F149 microcontroller with low power, GPS positioning system and GPRS bidirectional wireless transmission technology. On this basis, real-time monitoring and management for the maintenance of communications lines in grass-roots maneuvering affiliations on the condition of scattering conditions can be realized. This system has multiple monitoring and management functions including monitoring maintenance workers and communication line statuses as well as maintaining statistics data and operation processes. The system runs well in operation and its indexes are able to live up to the expected goals.
\end{abstract}

\section{Introduction}

According to special management requirements for dispersed maintenance workers in grass-roots maneuvering affiliations in maintenance of communications lines, this paper develops a remote monitoring system for managing maintenance information of communication lines. This system can be applied by grass-roots maneuvering affiliations in maintaining communications lines.

The system designed is characterized by stable performance, portable carrying and easy operation, and it is able to construct an integrate management platform for monitoring maintenance information by using the microcontrollers with low cost and power consumption, GPS positioning system, and GPRS bidirectional wireless transmission technology. Given the cases that the communication lines in the charge of grass-roots maneuvering affiliation are widely distributed and therefore maintenance workers are dispersed, this system can monitor the maintenance statuses of communication lines. In this way, it can real-timely acquire the locations, attendance rates and workloads of dispersed workers for maintaining communication lines as well as line conditions. Based on the acquired information, management department can have access to the detailed maintenance data of dispersed maintenance workers so as to timely find out various problems in the maintenance of lines to finally improve the maintenance quality of communication lines [1][2][3].

This developed system is put forward aiming at dispersed maintenance workers from special grass-roots affiliations, working positions and work assignment. It focuses on practicability with favorable functions and low price, thus showing advantages in application and popularization.

\section{Main body and functions of the system}

Main body of the system. This designed system is mainly composed of an information monitoring center, an information transmission network and a hand-held terminal (HHT). Thereinto, the information monitoring center includes a computer, an information receiving/transmitting equipment, system software (electronic map, database and serial communication programming) which are programmed using VC language, etc. The information transmission network transmits information by a short messaging service (SMS) of GSM of China Mobile or China Unicom. While the HHT 
primarily consists of a GPS receiver module, a single chip microcomputer control unit, and a GSM radio frequency transceiver module[4][5][6].

Main functions of the system. The monitoring center of the system real-timely collects the location information of the maintenance workers who are from grass-roots affiliations and are engaged in maintaining dispersed communication lines. Afterwards, the center automatically introduces the acquired information on the electronic map. If necessary, this center can analyze, compare and summarize the maintenance conditions of dispersed communication lines maintained by the workers, and timely process all sorts of feedback information.

Based on the according requirements, the information monitoring center will automatically generate data reports for each HHT so as to classify and collect the daily, weekly or monthly information including maintenance lengths and the number of markstones for communication lines as well as some other factors.

The system terminal sends codes containing 10 kinds of special commands which are utilized to report various special conditions which are possibly experienced by maintenance worker from grass-roots affiliations in the maintenance of dispersed communication lines[7].

\section{Terminal hardwares of the system}

The hardwares of the designed HHT equipment are comprised of a main control module (MSP430F149), a GPS module (JP13), a short message transceiver control module (BENQ M32), a FLASH memory, a LED display, antennae, etc, as shown in Figure 1.

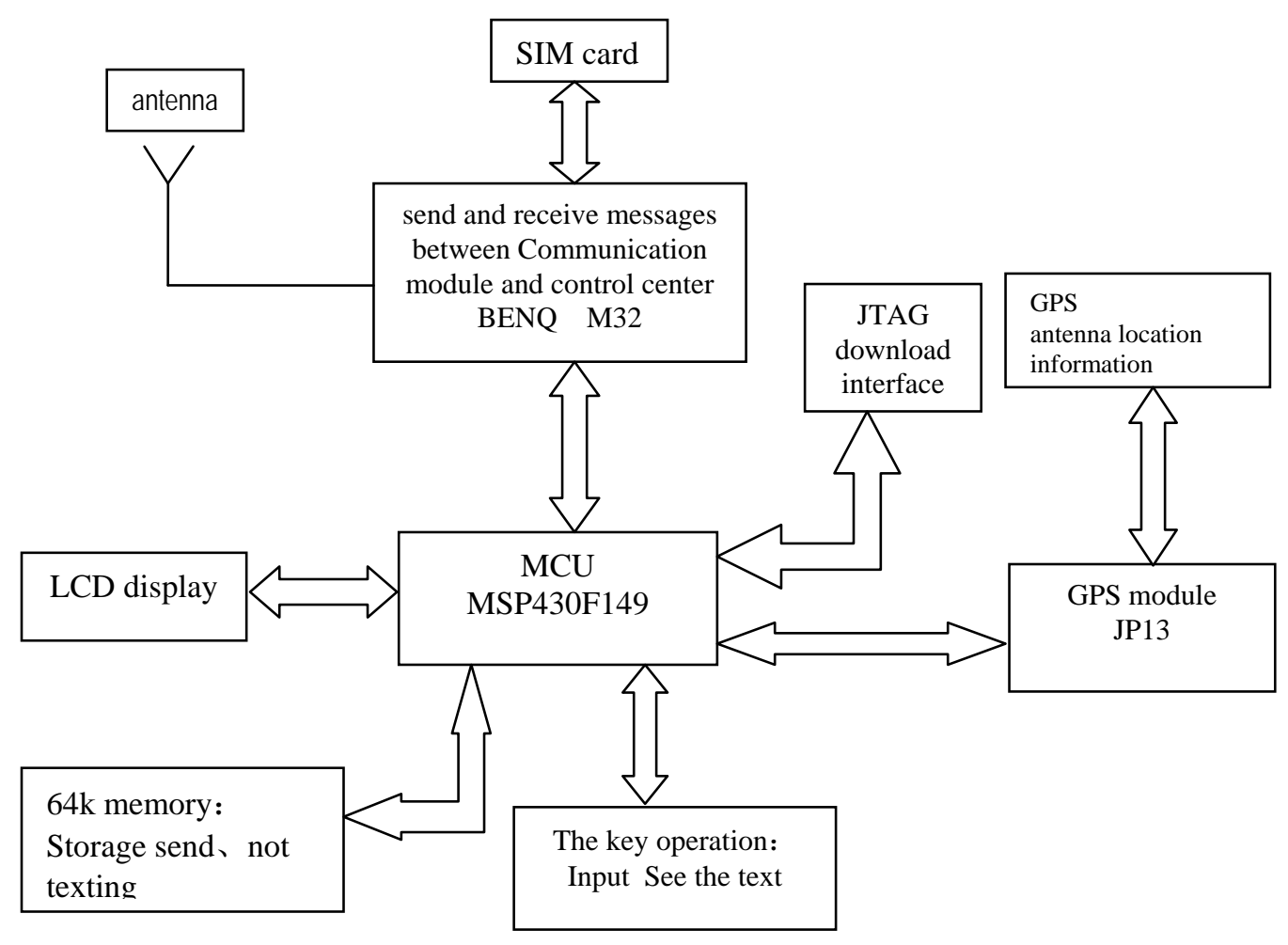

Fig.1 Circuit diagram of handheld terminal

The main control module and starting process of the terminal. A FLASH microcontroller (MSP430F149) is adopted as the main control module to uniformly control the functions of the HHT. With Von Neumann architecture, the MSP430F149 is a Flash microcontroller with 16-bit reduced instruction set computing (RISC), which consumes ultra-low power. Read-only memory (ROM), random access memory(RAM) and all periphery modules are located in the same address space. Two serial communication interfaces can be used as universal asynchronous receiver/transmitters (UART) 
or serial peripheral interfaces (SPI) so as to be applied to construct portable application system with strong functions.

The main control module is used to control the whole operation of the system. For example, it can coordinate the operations of GSM and GPS modules, and can code and send positioning information according to operation process. Meanwhile, it can perform power-on self test (POST) for each module by processing keyboard inputs, the display of characters on the liquid crystal panel, state display of indicator lights and generation of key tones.

As the system is powered on, the BENQ-M32 module, the GPS module (JP13) and the LED display are initialized. English and Chinese characters are preseted to send data to the LED display so as to present the boot information. Instructions are sent to the BENQ-M32 module to detect the SIM card and the strength of GSM signals. The GPS module (JP13) receives GPS positioning information which are output on the LED display. When the above detections are finished, the terminal automatically sends boot information to the information center, showing that this terminal has logged in the system.

In the absence of SIM card being detected, an error message appears and the program is interrupted so that the terminal will not work. If the SIM is found to be normal, the operation continues.

The HHT reports the position information through automatic timing or manual modes. Furthermore, the information center of the system sends automatic transmitting commands to the terminal by way of GSM short messages, and then the BENQ-M32 receives the information and transfers it to the MSP430F149 microcontroller. The MSP430F149 processes the information and obtains the intervals of automatic transmitting in a range of $0-255$ min ( 0 presents the manual process). At this time, the terminal enters into the automatically transmitting state. The MSP430F149 microcontroller formats the terminal number, information contents, latitudes and longitude coordinates and transmission time into character strings which are sent to BENQ-M32. Afterwards, the BENQ-M32 sends the information to the information center of the system by way of short messages.

GPS module and information collection of the terminal. The GPS module group JP13 (Germany FALCOM Company) equipped with the SiRF Star III chipset is used as the GPS module to process the positioning information. The JP13 is mainly characterized by high positioning accuracy with an error less than $10 \mathrm{~m}$, fast positioning speed with cold-start speed less than $30 \mathrm{~s}$, ultrahigh sensitivity reaching $-159 \mathrm{dbm}$. Moreover, it consumes little power. There is merely $105 \mathrm{mw}$ of power being consumed when the working voltage is $+3.3 \mathrm{~V}$ DC. The JP13 outputs the received positioning information in NMEA-0183 format to the MSP430F149 through serial ports with a baud rate of 115,200 .

When receivers in running receive and calculate the GPS positioning information and then send the information to the UART serial ports of the MSP430F149. And then, the ports extract the positioning information based on the data frame structure including frame head, intra-frame data and tail frame. Information is processed based on the time and geographic coordinates extracted from “\$GPRMC” frame data instead of "\$GPGGA", "\$GPGSA” and “\$GPGSV”.

Data transmission and indicator light control. The SMS of GSM is applied as the platform to transmit information so as to guarantee the transmission of remote data and the communication of monitoring equipment. This is characterized by bidirectional wireless data transmission, stable performance, little limitation to the number of users and no need for further network being set. Short messages are received and sent using the BENQ-M32 module developed by the BenQ Corporation with internal antennae and supporting double frequencies of 900/1800MHz. This BENQ-M32 module is used to receive and send short messages and detect SIM cards. Besides, it transfers the received messages to the microcontroller to be processed so as to send out the message from the microcontroller. The microcontroller directly sends AT commands to the GSM modules through UART serial ports to further receive and dispatch, search and management of the SMS based on GSM. 
Three indicator lights are designed to show the normal operating states: LED 1 shows the GPS state. In the extraction of GPS signals, this light flickers while being off under normal operation. This light is controlled by the MSP430F149 microcontroller as P54 outputs pulse to make LED1 flash. LED2 indicates the GSM signals. It does not work when there are no signals; while when signals are found normally, it flickers. This light is under the control of the GSM module though P46 output. LED3 is a charging indicator and flashes in the charging process. After the charging process is finished, this light will automatically put out.

\section{Design of system application software}

Key points of monitoring central. The monitoring center is a software with Chinese language developed using Visual C++ 6.0 based on Microsoft Windows XP system. Terminal software is embedded and developed using $\mathrm{C}$ programming language. The software is programmed on the operation system and connected with the SEM transceiver module through RS-232 interface with the rate of data transmission of 38,400 bps.

The programming is conducted using several technologies including database and digital electronic map. A digital electronic vector map with a scale of 1:25,000 is applied to show the routes of communication lines and the real-time tracks of maintenance workers when they maintain the dispersed communication lines. The map can be magnified, reduced and roamed to hierarchically display the tracks. Moreover, by searching database, we can compare the coordinates of original paths inputted in advance to reveal whether maintenance workers maintain communication lines along the line routes. Furthermore, the maintenance speed for communication lines can be calculated according to the intervals of reported data and marching distances so as to determine the maintenance modes, namely, by bus or on foot.

Realization of application software. The SMS specifications formulated by ESTI that are associated with the transceiver of short messages are shown as follows. GSM 03.38 and GSM 03.40 focus on the technological realization (including coding schemes) of SMS; these specifications specify the DET-DCE interface standard for SMS, namely, attention (AT) command set, as shown in Table 1.

Tab.1 The table of AT command set

\begin{tabular}{|l|l|l|l|l|}
\hline AT command & functions & AT command & functions \\
\hline AT+CMGC & Send an SMS command & AT+CNMI & New SMS message indications \\
\hline AT+CMGD & Delete SMS message & AT+CPMS & Preferred SMS message storage \\
\hline AT+CMGF & Select SMS message format & AT+CSCA & SMS service center address \\
\hline AT+CMGR & Read SMS message & AT+CSCB & Select cell broadcast messages \\
\hline AT+CMGS & Send SMS message & AT+CSMP & Set SMS text mode parameters \\
\hline AT+CMGW & Write SMS message to memory & AT+CSMS & Select Message Service \\
\hline AT+CMSS & Send SMS message from storage & \multicolumn{1}{|l|}{} \\
\hline AT+CMGL & $\begin{array}{l}\text { List SMS message from preferred store (PDU/text: 0/“REC UNREAD”, 1/“REC READ”, } \\
\text { 2/“STO UNSENT”, 3/“STO SENT”, 4/“ALL”) }\end{array}$ \\
\hline
\end{tabular}

In China, SMS messages are sent and received through a PDU mode. The PDU mode supports all telephone terminals. It can not only use any character sets, but also can normally adopt default coding mode.

According to the specification of GSM 07.05, the controller can read the received short messages under the PDU mode when it sends AT commands to the GSM module through UART interface. In the case of $A T+C M G F=0$, the controller is set under the PDU mode; while when $A T+C M G R=1$, the controller reads one of the received messages. Under the PDU mode, the GSM communication module answers in a format as: +CMGR: $<$ stat $>,<$ length $><\mathrm{CR}><\mathrm{LF}><$ pdu $>$. Stat presents the state of this short message and is generally set as 0 or 1.0 suggests that this message is new and not read while 1 shows that it has been read. Length is a decimal number, indicating the length of the PDU data minus the address length of the SMS center (SEMC) with unit of byte. $<\mathrm{CR}><\mathrm{LF}>$ presents carriage 
return and line feed. pdu is the data packet under the PDU mode. By analyzing the data packet under the PDU, the received information is analyzed based on the data format under the PDU.

The data packet under the PDU mode is composed of SMSC address and TPDU, in which one data unit represents one byte. The numbers of the SEMS and transmitting source, date and time are represented by binary-coded decimal (BCD) codes with low bit ahead followed by high bit.

Short messages in English under the PDU mode are coded using seven bits in the GSM character set with TP-data-coding-scheme (TP-DCS) value of 00. If this message is composed of English characters "Hello!" each character is converted to seven-bit ASCII codes. The low bit of the following characters are regulated ahead to make up the difference of first characters. The hexdecimal number coded based on the character string of "Hello!" is shown in Figure 2.

Multiple special command codes are preset in the information center of the system to be used to define and interpret the meanings of the information sent by the terminals. In this way, the special situations encountered by the dispersed maintenance workers in maintenance can be timely reflected. These situations include normal markstone positioning, constructions being found in maintenance, outcropped optical cables, damaged pole lines, and the displacement and lose of markstones.
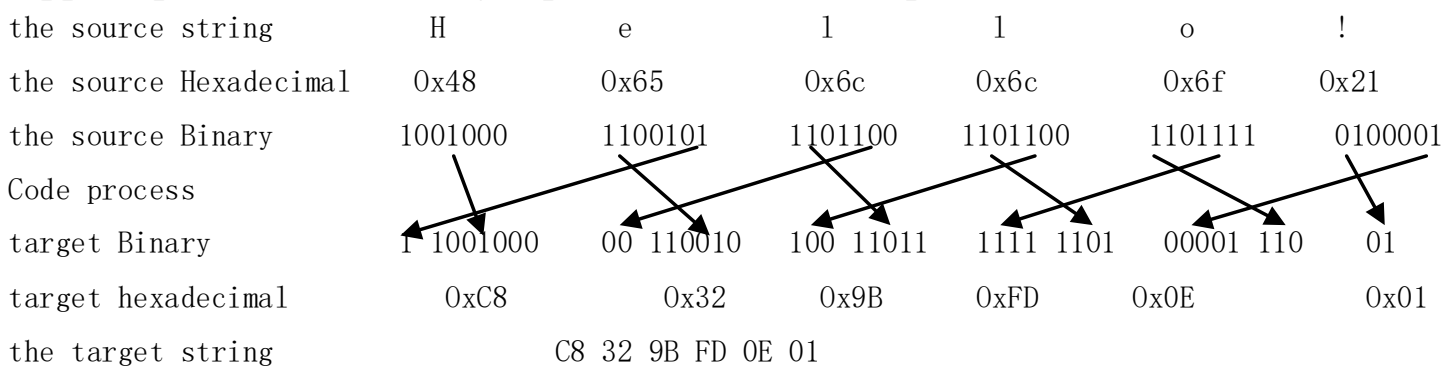

Fig. 2 Hexadecimal number of string coded

The information center automatically reads the information in SIM. Through the analysis of the information, the data are plotted on the map based on corresponding international standard to real-timely show the marching tracks of current maintenance workers. The system is endowed with multiple data statistics functions. For example, the system can search corresponding information as required and show the marching track of certain maintenance workers at certain stage. In addition, the system can classify and collect data reports, formulate maintenance plans and evaluate maintenance results according to plan.

\section{Conclusions}

Repeated experiments show that the developed management system for monitoring the information on the maintenance of maneuver communication lines can accurately perform positioning and icon plotting. Moreover, it is characterized by reliable communication, stable running, low power consumption and easy operation. It reaches the design requirements in functions and the indexes of the system technology are shown to meet the expected goals. The indexes including environmental adaptability, electromagnetic compatibility and security are proven to be normal. This system can significantly reduce the cost in human, financial and material resources cost by maintenance workers from grassroots affiliations for maintaining dispersed communication lines, thus improving the informatization level.

\section{Acknowledgement}

In this paper, the research was sponsored by the nature science research projects of education department in Shaanxi Province government and key project of education department in Shaanxi provincial government (Project No. 13BZ69). 


\section{References}

[1] Zhang Hongbo,Zhao Donglai,Wang Yang,etc. Analysis on impacts of sandstorm on electric power optical communication cable lines[J].stduy on optical communication,2013,12(180):39-41.

[2] Chen Haihong,Zhang Jing,Wan Shuting.Development and application of electric power communication line management system based on GIS[J].Proceedings of the CSU-EPSA, 2013,4(25):26-30.

[3] Meng Xiaoxin,Chen Hongsheng,Li Jun,etc.Commnication lines maintenance system based on WebGIS [J].computer engineering,2008,9(34):248:250.

[4] Ge Yurong,Li Yanjiang,Wang Xuzhu.Failure monitor system design for communication circuit [J].Computer measurement \& control,2006,(01).

[5] Han Hui,Cao Bangqin,Ni rongjun.Design and realization of infrared communication device based on MSP430[J].bulletin of science and technology,2014,11(30):72-74.

[6] Zhang Wangxian,Yao Tongtong,Zhao Jiurui,etc.The design of simple DC electronic load based on MSP430[J].research and exploration in laboratory,2015,8(34):77-80.

[7] Cui Ming,Tong Gang,Wu Zhiyong. The intensity test system for compression resistance of paper based on MSP430[J].microcomputer information,2007,10(23):4-5. 